AperTO - Archivio Istituzionale Open Access dell'Università di Torino

\title{
Genome-wide analysis of cell wall-related genes in Tuber melanosporum
}

\section{This is the author's manuscript}

Original Citation:

Availability:

This version is available http://hdl.handle.net/2318/102720

since 2016-09-28T11:51:45Z

Published version:

DOI:10.1007/s00294-012-0374-6

Terms of use:

Open Access

Anyone can freely access the full text of works made available as "Open Access". Works made available under a Creative Commons license can be used according to the terms and conditions of said license. Use of all other works requires consent of the right holder (author or publisher) if not exempted from copyright protection by the applicable law. 
This is the author's final version of the contribution published as:

R. Balestrini; F. Sillo; A. Kohler; G. Schneider; A. Faccio; E. Tisserant; F. Martin; P. Bonfante. Genome-wide analysis of cell wall-related genes in Tuber melanosporum. CURRENT GENETICS. 58(3) pp: 165-177. DOI: $10.1007 / \mathrm{s} 00294-012-0374-6$

The publisher's version is available at:

http://www.springerlink.com/index/pdf/10.1007/s00294-012-0374-6

When citing, please refer to the published version.

Link to this full text:

http://hdl.handle.net/2318/102720 


\title{
Genome-wide analysis of cell wall-related genes in Tuber melanosporum
}

\author{
Raffaella Balestrini1, 2
}

Fabiano Sillo 1, 2

Annegret Kohler 3

Georg Schneider 4

Antonella Faccio 1, 2

Emilie Tisserant 3

Francis Martin 3

Paola Bonfante 1, 2

1.Istituto per la Protezione delle Piante del CNR, UOS TorinoItaly

2.Dipartimento di Biologia VegetaleUniTOItaly

3.UMR 1136, INRA-Nancy Université, Interactions Arbres/MicroorganismesFrance

4.Bioinformatics InstituteA*StarSingapore

DOI: $10.1007 / \mathrm{s} 00294-012-0374-6$

Cite this article as:

Balestrini, R., Sillo, F., Kohler, A. et al. Curr Genet (2012) 58: 165. doi:10.1007/s00294012-0374-6
Abstract
A genome-wide inventory of proteins involved in cell wall synthesis and remodeling has been obtained by taking advantage of the recently released genome sequence of the ectomycorrhizal Tuber melanosporum black truffle. Genes that encode cell wall biosynthetic enzymes, enzymes involved in cell wall polysaccharide synthesis or modification, GPI-anchored proteins and other cell wall proteins were identified in the black truffle genome. As a second step, array data were validated and the symbiotic stage was chosen as the main focus. Quantitative RT-PCR experiments were performed on 29 selected genes to verify their expression during ectomycorrhizal formation. The results confirmed the array data, and this suggests that cell wall-related genes are required for morphogenetic transition from mycelium growth to the ectomycorrhizal branched hyphae. Labeling experiments were also performed on T. melanosporum mycelium and ectomycorrhizae to localize cell wall components. 


\section{Keywords}

Tuber melanosporumFungal genomeCell wallEctomycorrhizaeSymbiotic interactions

Communicated by B. Cormack.

R. Balestrini and F. Sillo equally contributed to the paper.

\section{Electronic supplementary material}

The online version of this article (doi:10.1007/s00294-012-0374-6) contains supplementary material, which is available to authorized users.

\section{Introduction}

The fungal cell wall is a dynamic structure that protects the cell from changes in osmotic pressure and other environmental stress, while allowing it to interact with the environment and other organisms. In addition to maintaining cell shape and integrity during environmental stress, the cell wall is also involved in the interaction with its surroundings and serves as a signaling center to activate signal transduction pathways within fungal cells (Bowman and Free 2006). The fungal cell wall is usually composed of chitin, glucans and proteins that are extensively cross-linked together to form a complex network, which forms the structural basis of the cell wall (Bowman and Free 2006). However, the composition varies from taxonomic group to taxonomic group and may also vary within a single fungal isolate, depending on the conditions and the stage of growth. A cell wall in fact undergoes constant changes, i.e., during cell expansion and division in yeasts, and during spore germination, branching and septum formation in filamentous fungi. The formation and remodeling of the fungal cell wall involves numerous biosynthetic pathways and the concerted actions of many gene products (Bowman and Free 2006). Although the yeast cell wall structure and assembly is considered as a general model (Lesage and Bussey 2006), recent results, derived from wholegenome analysis of other fungi, have highlighted a number of differences between filamentous fungi and yeast.

Truffles, as symbiotic ectomycorrhizal fungi, have a complex life cycle that is characterized by three stages: vegetative growth as filamentous mycelium; the formation of ectomycorrhizae (ECM), which requires mutual recognition between the fungus and the host plant; the production of reproductive carpophores containing sexual spores. The transition from the vegetative mycelium to the symbiotic and/or reproductive stage requires morphogenetic processes to pass from an apical growth to the formation of fungal tissue-like structures (Balestrini et al. 1996, 2000). In this context, cell wall-related and secreted proteins are of special interest, since they may play a role in a number of specific processes. During interaction with plants, the fungal wall is the first part to come into contact with the host cells, and it plays a role in cell-to-cell adhesion. In addition to the contact with host surface, the fungal cell wall of ECM fungi is involved in both the adhesion events that occur during the mantle formation and in the creation of the symbiotic interface between the Hartig net hyphae and the root cells (Balestrini et al. 2011). The involvement of cell wall components during ECM development has already been investigated (Balestrini et al. 1996; Laurent et al. 1999; Martin et al. 1999; Tagu et al. 2001). Taking advantage of the recently released genome sequence of the Tuber melanosporum black truffle (Martin et al. 2010), we have obtained a genome-wide inventory of the proteins that are involved in cell wall synthesis and remodeling. For this purpose, we searched for genes that encode cell wall biosynthetic enzymes, cell wall polysaccharide enzymes, glycosylphosphatidylinositol (GPI)-anchored proteins and other cell wall proteins. 
Prediction tools were used, in combination with phylogeny reconstruction and expression analyses, to characterize almost 100 of these genes. Validation experiments were performed on some selected genes, focusing on the expression in ECM versus free-living mycelium (FLM). Furthermore, labeling experiments were performed on FLM and ECM to localize cell wall components.

\section{Materials and methods}

\section{Biological materials}

The T. melanosporum mycelium (Tmel28 strain) was grown in a liquid Modified Melin_Norkrams (MMN) medium in a dark room at $25{ }^{\circ} \mathrm{C}$ for 1 month before harvesting. The fully developed $T$. melanosporum ectomycorrhizae (ECMs; Martin et al. 2010) were sampled from mycorrhized hazelnut plants (Corylus avellana L.) that were produced as described in Martin et al. (2010) and grown in the AGRI-TRUFFE greenhouse facilities (Saint-Maixant, France).

\section{Morphological analysis}

The T. melanosporum mycelium and ECM paraffin sections (Martin et al. 2010) were stained with fluorescein isothiocyanate-conjugated wheat germ agglutinin (FITC-WGA; $10 \mathrm{mg} / \mathrm{ml}$ ) to localize chitin. The stained sections were observed using a Leica TCS SP2 confocal microscope. An excitation wavelength of $488 \mathrm{~nm}$ and an emission window of 500-520 nm were used to visualize the FITC. Immunogold experiments were performed, as described in Balestrini et al. (1996), to localize glucans. A monoclonal antibody anti- $\beta$-1,3-glucan (Biosupplies, Australia) was used at a 1:100 dilution, while the polyclonal antibody anti- $\beta$-1,6-glucans (kindly provided by Laura Popolo; Montijn et al. 1999) was used at a 1:10 dilution. The primary antibody was omitted for the negative control; reactions were performed on yeast with the anti- $\beta-1,6$-glucan as the positive control.

\section{Bioinformatics}

The gene models (putative genes) encoding proteins involved in cell wall synthesis/degradation were identified at the TuberDB Tuber genome database (http://mycor.nancy.inra.fr/IMGC/TuberGenome/). Searches were also performed using a range of sequences related to fungal cell wall proteins and genes available from fungi in order to probe the Tuber genome database using BLAST algorithms. All the detected gene models were inspected manually, and the automatically selected best gene model of the TuberDB database was modified, if necessary. The detected putative homologs were characterized on the basis of the conserved domains, identities and $E$ values. Manual annotation was carried out using Artemis software (http://www.sanger.ac.uk/Software/Artemis/). Further information about the name and structure of the genes was obtained using BLASTP, which is available on NCBI (http://www.ncbi.nlm.nih.gov/) and EMBL (http://www.ebi.ac.uk/Tools/blastall/index.html). Each validated homolog was also used for a BLAST search at http://mycor.nancy.inra.fr/IMGC/TuberGenome/blast.php, which has a database with five reference Ascomycota: Saccharomyces cerevisiae, Neurospora crassa, Magnaporthe grisea, Aspergillus nidulans and Botrytis cinerea. The protein sequences deduced from the present genome survey were used for extensive database searches for both homolog sequences and sequences that were closely related phylogenetically, and a multiple protein alignment was constructed using the MUSCLE software (Edgar 2004). Phylogenetic trees were constructed with the maximum likelihood method, using the MEGA software, Version 5.0 (Tamura et al. 2011). Bootstrap analyses were conducted on the basis of 1,000 re-samplings of the sequence alignment. 
Taking the predicted T. melanosporum Genoscope gene models (7,496 predicted proteins) as input, putative GPI proteins were identified by means of a selective fungal-specific algorithm named BigPI (Eisenhaber et al. 2004) which was used for C-terminal GPI modification site prediction. The GPI-anchor predictor is available online at the Web site of the Institute of Molecular Pathology (IMP) (http://mendel.imp.ac.at/gpi/gpi_server.html). Sequences with a predicted modification site were further analyzed using SignalP (Bendtsen et al. 2004) to ensure that they also contain a signal peptide. To check for possible transmembrane domains in the mature proteins, the resulting set was then analyzed with the ANNIE protein sequence annotation and interpretation environment (Ooi et al. 2009) which includes HMMTOP (Tusnády and Simon 2001), DAS-TMfilter (Cserzo et al. 2004), Phobius (Käll et al. 2004) and SAPS (Brendel et al. 1992) as predictors for transmembrane segments.

\section{Transcriptome analyses}

The T. melanosporum custom-exon expression array $(4 \times 72 \mathrm{~K})$ manufactured by Roche NimbleGen Systems Limited (Madison, WI) contained five independent, non-identical, 60-mer probes per gene model coding sequence. A total of 7,496 annotated protein-coding gene models, 5,736 TE sequences, 3,913 random 60-mer control probes and labeling controls were included in the array. For 1,876 gene models, technical duplicates were included in the array. Samples used in this study were obtained from FLM, fruiting bodies (FB) and ectomycorrhizae (ECM) as described previously (Martin et al. 2010). Total RNA preparations (four biological replicates for ECM, five for FB and seven for FLM) were amplified using the SMART PCR cDNA Synthesis Kit (Clontech) according to the manufacturer's instructions.

Single dye labeling of samples, hybridization procedures, data acquisition, background correction and normalization were performed at the NimbleGen (NimbleGen Systems, Reykjavik, Iceland), following their standard protocol.

Raw array data were filtered for non-specific probes (a probe was considered as non-specific if it shared more than $90 \%$ homology with a gene model other than the gene model it was made for) and renormalized using the ARRAYSTAR software (DNASTAR, Inc., Madison, WI, USA). For 1,015 gene models, no reliable probe was left. A transcript was deemed to be expressed when its signal intensity was threefold higher than the mean signal-to-noise threshold (cutoff value) of 3,913 random oligonucleotide probes present on the array (50-100 arbitrary units). Gene models with an expression value higher than threefold the cutoff level were considered as transcribed. A Student $t$ test with false discovery rate (FDR) (Benjamini-Hochberg) multiple testing correction was applied to the data using the ARRAYSTAR software (DNASTAR). Transcripts with a significant $P$ value $(<0.05)$ and $\geq 4$-fold change in transcript level were considered as differentially expressed. The complete expression data set is available as series (accession number GSE17529) at the Gene Expression Omnibus at NCBI (http://www.ncbi.nlm.nih.gov/geo/).

Expression levels of the annotated genes were also detected by massive cDNA sequencing of the abovementioned FLM, ECM and FB libraries with the Solexa/Illumina technology. Deep sequencing was carried out at the Genoscope facilities as described in Denoeud et al. (2008) and mapped to the T. melanosporum genome and gene models, as reported by Martin et al. (2010). The single-end reads obtained were 36 nucleotides long and were deposited in the Tuber Gbrowse (http://mycor.nancy.inra.fr/cgi-bin/secure/gbrowse/). Data were normalized according to sample size and gene length and reported in RPKM (Reads Per Kilobases of exon model per Million mapped reads). 


\section{RNA extraction, cDNA synthesis and quantitative realtime PCR}

The Mel28 T. melanosporum strain cultures and T. melanosporum/Corylus avellana ECMs were used to extract the total RNA. The RNA was extracted using the 'pine tree method' (Chang et al. 1993), for both mycelium and ECM. Genomic DNA was removed using a Turbo DNA-free ${ }^{\mathrm{TM}}$ reagent (Ambion, Austin, TX, USA), according to the manufacturer's instructions. The absence of genomic DNA was verified through retro-transcription PCR (RT-PCR, OneStep RT-PCR, Qiagen) with specific primers for the CHI gene (TmelCHIf and TmelCHIr) designed to amplify the intron. Five hundred nanograms of total RNA was used for each sample to synthesize the cDNA, according to the SuperScriptII ${ }^{\circledR}$ Reverse Transcriptase (Invitrogen) procedure. qRT-PCR primers were designed using PerlPrimer, a free, open-source GUI application (http://perlprimer.sourceforge.net/). Before qRT-PCR, the primers were tested on genomic DNA extracted from the T. melanosporum mycelium (positive control) and C. avellana leaves (negative control) with the Dneasy Plant Mini Kit (Qiagen). The specific primer sequences are shown in Table S1.

Quantitative RT-PCR was carried out with StepOne apparatus (Applied Biosystem), according to the procedure described in Zampieri et al. (2011). The baseline range and $C_{\mathrm{T}}$ values were automatically calculated using the StepOne software. The expression of candidate genes was normalized to that of Tmeltefl-beta (GSTUMT00001146001) by subtracting the $C_{\mathrm{T}}$ value of Tmeltefl-beta from the $C_{\mathrm{T}}$ value of the candidate gene resulting from the $\Delta C_{\mathrm{T}}$. The expression ratios were calculated without the PCR efficiency correction from equation $2 \Delta \Delta C$,

where $\Delta \Delta C_{\mathrm{T}}$ represents the $\Delta C_{\mathrm{T}}$ sample $-\Delta C_{\mathrm{T}}$ control. All the reactions were performed for at least two biological and three technical replicates. Statistical analyses were carried out using Rest2008, version 2.0.7, considering data with a $P$ value $<0.05$ as being significantly different.

\section{Results}

\section{Detection of cell wall components on the cell surface}

Labeling experiments were performed to localize cell wall polysaccharide components in $T$. melanosporum. As expected, immunofluorescence, using a WGA-FITC complex to localize chitin, led to a green signal on the T. melanosporum cell walls on the fungal hyphae, both in the pure culture and in the ECM stage (not shown). Immunogold experiments allowed us to verify the presence of $\beta$-1,3-glucans (Fig. 1) in the electron-transparent layer in the two considered life cycle stages, while no labeling was evident for $\beta-1,6$ glucans (Fig. 2). 

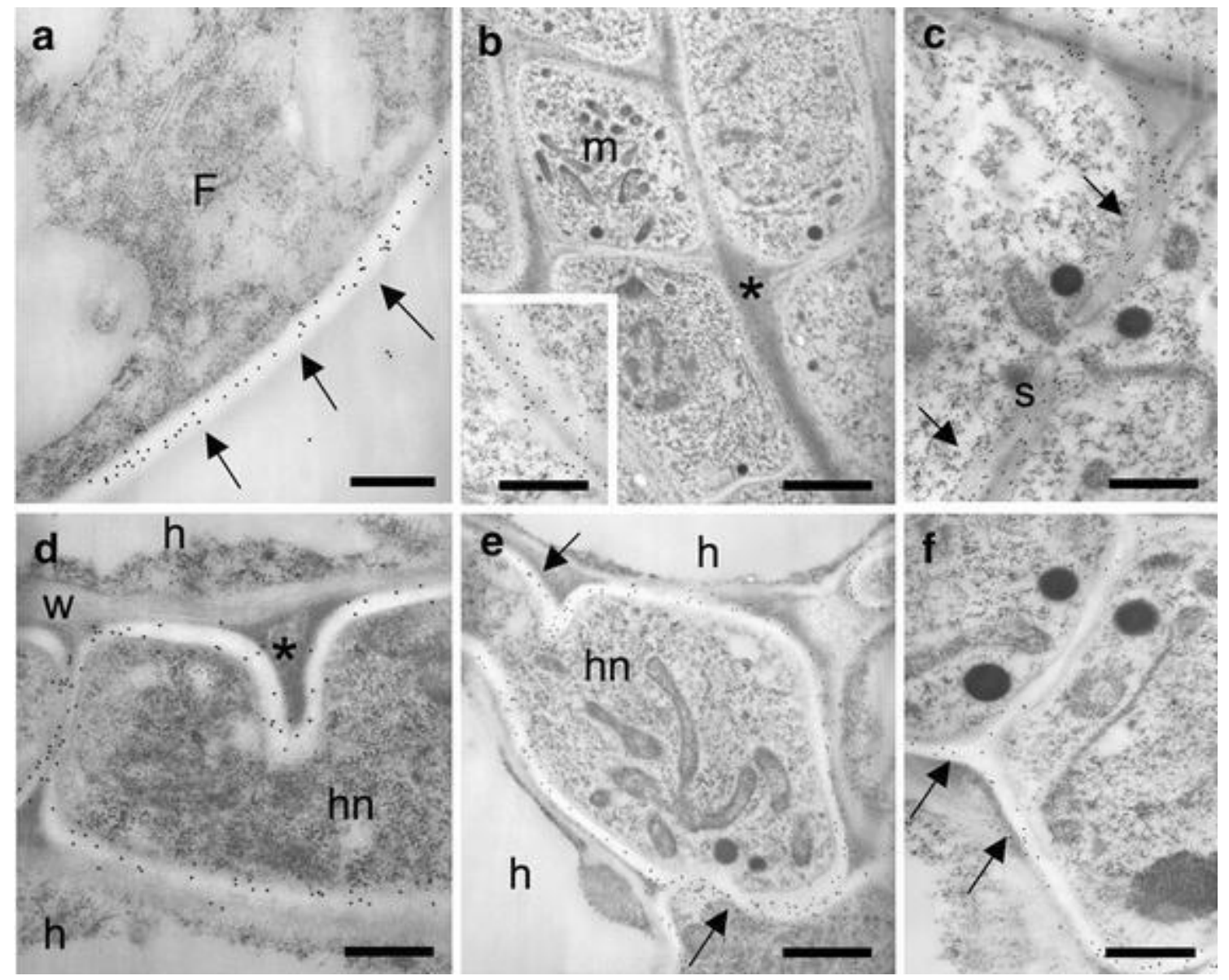

Fig. 1

Immunogold localization of $\beta$-1,3-glucans on thin sections from mycelium grown in pure culture (a) and from T. melanosporum/C. avellana ectomycorrhizae (b-f). a The arrows point to $\beta-1,3-$ glucans-associated gold granules accumulated in the electron-transparent cell wall layer. $\mathbf{b}$ Gold granules are present on the cell wall electron-transparent layer of the hyphae that form the mantle, while no labeling is evident on the cementing material present among the hyphae (asterisk). Inset Magnification of a cell wall region. c Magnification of a septum in the mantle region. $\mathbf{d}-\mathbf{f}$ Hartig net region: cell wall labeling of hyphae progressing between the root cells during the Hartig net establishment. Gold granules are present on the fungal cell wall (arrows), while no labeling occurs on the host cell walls. No gold granules are present on the electron-dense material with a triangle shape, which fills the interhyphal space (asterisk). $h$ host cell, $h n$ Hartig net, $m$ mantle, $n$ nucleus, $s$ septum, $F$ fungal hypha, $w$ plant cell wall. Bars correspond to $0.48 \mu \mathrm{m}$ in $\mathbf{c}, \mathbf{f}$ and inset, $0,35 \mu \mathrm{m}$ in a and $\mathbf{b}, 0,26 \mu \mathrm{m} \mathbf{d}, 0,84 \mu \mathrm{m}$ in $\mathbf{e}$ 

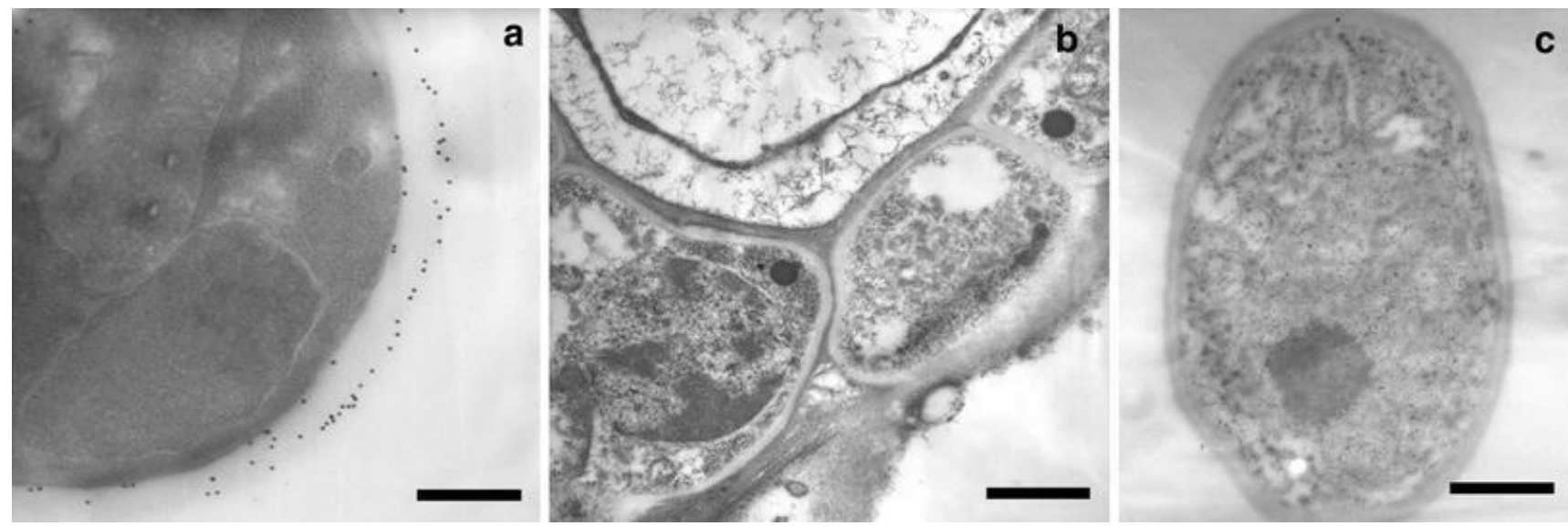

Fig. 2

Immunogold labeling of $\beta$-1,6-glucans on thin sections of yeast (a) and T. melanosporum ectomycorrhizae (b) and mycelium grown in pure culture (c). a Specific labeling (arrows) of the yeast cell wall after treatment with the anti- $\beta-1,6$-glucans antibody. No specific labeling could be observed on the truffle cell walls in either symbiotic (b) or free-living stage (c). $Y$ yeast cell, $F T$. melanosporum hyphae. Bars correspond to $0.26 \mu \mathrm{m}$ in $\mathbf{a}$ and $0.85 \mu \mathrm{m}$ in $\mathbf{b}$ and $\mathbf{c}$

\section{Gene catalog of cell wall metabolism}

In silico analysis of the T. melanosporum genome led to the identification of the main enzymes and regulators involved in fungal cell wall dynamics, and allowed the identification of several members of different gene families (Table S2). As reported in Table S2, eight gene models were modified. We identified genes belonging to the following main groups: chitin synthases and chitinases, $\beta-1,3-$ glucan synthases, $\alpha-1,3$-glucan synthases, enzymes involved in the $\beta$-1,6-linked glucan synthesis, glucanases and glycosyl transferases, hydrophobins and proteins involved in mannoprotein biosynthesis (e.g., MNN9 complex proteins) as well as enzymes involved in the processing of cell wall components (e.g., mannosidase DCW1-like, putative chitin deacetylases). Furthermore, small secreted proteins (SSPs), e.g., TmelPLA2 and TmelCFEM, were found in the T. melanosporum genome and annotated (Table S2).

\section{T. melanosporum genes involved in chitin metabolism}

Chitin is a linear polymer of $\beta$-1,4-linked GlcNAc. Chitin synthases (EC 2.4.1.16) belong to a gene family that can be divided into several classes with specific roles, as demonstrated in several fungi through genetic analyses (Bowman and Free 2006). Six genes coding for "standard" chitin synthases (TmelCHSD, TmelCHS2, TmelCHS3, TmelCHS4, TmelCHS7 and TmelCHSG) and two genes coding for myosin-chitin synthases (TmelCHS6 and TmelCHS8) were identified in $T$. melanosporum and, as revealed by phylogenetic analysis, all the CHS classes seem to be represented by at least one member in T. melanosporum, except class III for which two members are present (Fig. 3). The latter class is unique to filamentous fungi and is specifically involved in apical growth; a role for a class III CHS has already been demonstrated during spore cell wall formation in T. borchii (Borgia et al. 1996; Balestrini et al. 2000). The number of CHS genes in T. melanosporum is in accordance with that present in other filamentous fungi (Table S3; Choquer et al. 2004). Two of the newly identified CHSs (CHS6 and CHS8) have an N-terminal myosin motorlike domain (which is perhaps incomplete in TmelCHS6). This domain is only present in CHSs from filamentous fungi: the MMD (myosin motor domain) may allow hyphal tip-polarized chitin 
synthesis to take place via interaction with the actin cytoskeleton (Takeshita et al. 2006). The myosin-CHSs in Ustilago maydis and Fusarium oxysporum are critically involved in (and required for) polarized growth during plant infection (Weber et al. 2006; Martin-Urdiroz et al. 2008; Treitschke et al. 2010).

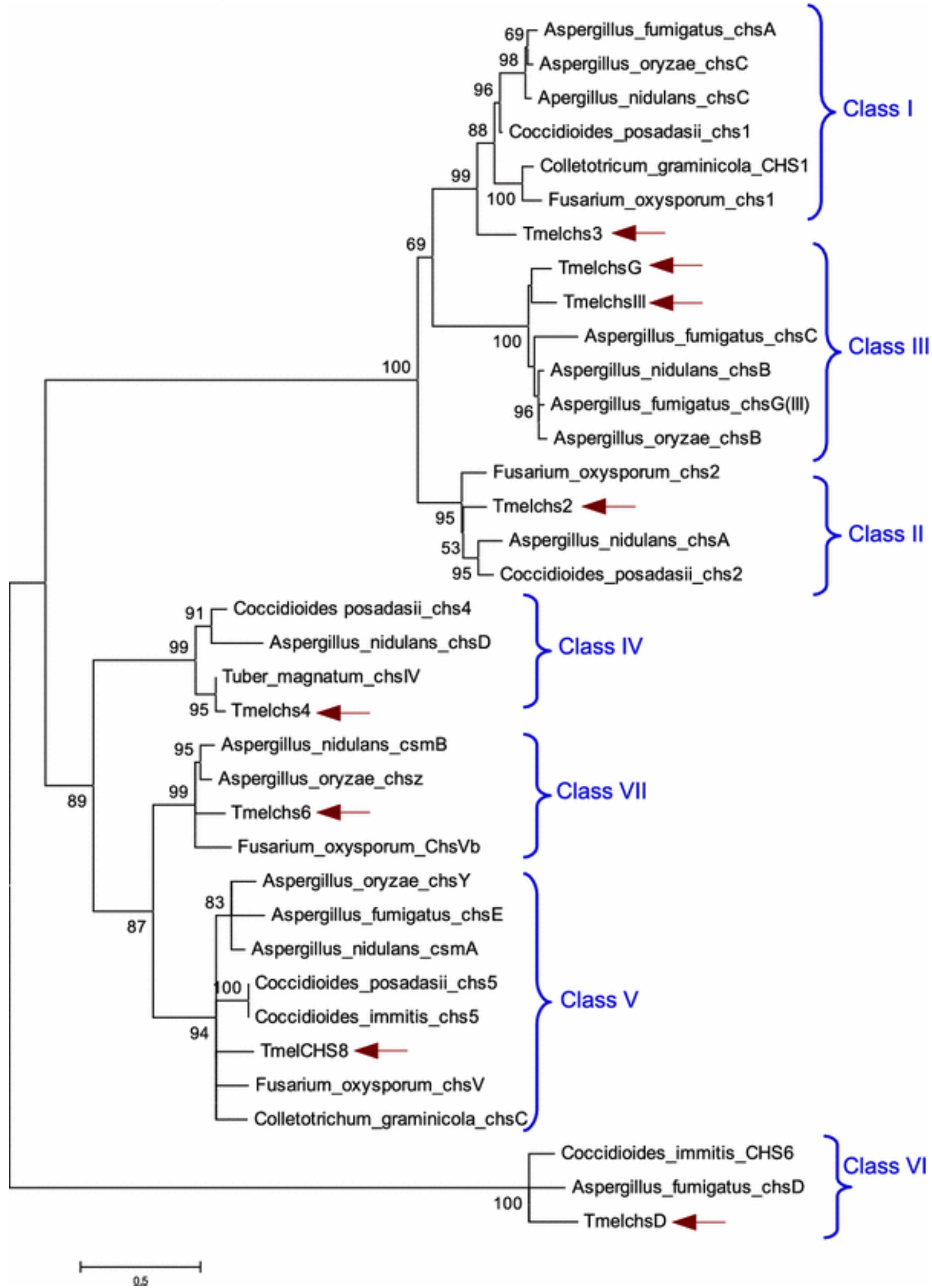

Fig. 3 
Phylogenetic relationships between filamentous Ascomycetes based on amino acid deduced sequences for the representative chitin synthase family. The sequences were aligned using Muscle and the unrooted tree was constructed using the maximum likelihood method. Numbers indicate bootstrap values, and are given only for $>50 \%$. Arrows point to the T. melanosporum sequences

Chitinases (EC 3.2.1.14) are chitin-degrading enzymes that hydrolyze the $\beta-(1,4)$ linkages of chitin. The fungal chitinases identified so far belong to glycoside hydrolase (GH) family 18 (Duo-Chuan 2006; Seidl 2008; Karlsson and Stenlid 2008) in the carbohydrate active enzyme (CAZy) classification (http://www.cazy.org/GH18.html; Cantarel et al. 2009) and can be phylogenetically divided into three different subgroups (A, B and C; Gruber et al. 2011). In recent years, additional chitinase genes have been discovered as a result of whole-genome sequencing projects, e.g., the presence of 5, 12, 19, 15, 20 and 20 genes that encode putative chitinases in Candida albicans, $N$. crassa, Gibberella zeae, Magnaporthe grisea, Aspergillus nidulans and Hypocrea jecorina, respectively (Karlsson and Stenlid 2008). The T. melanosporum genome only contains five chitinase genes (Table S2). As revealed by means of phylogenetic analysis, two of these genes belong to subgroup A and the other two to subgroup B (data not shown). An additional chitinase candidate gene has been found, and phylogenetic analysis has shown that it clusters with the subgroup B sequences. A chitinase active site (IPR001579: Glyco_hydro_18_chit_AS) has been identified in its sequence, by means of InterProScan analysis, while a signal peptide, predicted with SignalP and present in the other two group B chitinases, is not present.

\section{T. melanosporum genes involved in glucan metabolism}

As in other filamentous fungi, we found a single gene coding for $(1,3)-\beta$-glucan synthase (TmelFKSA) in T. melanosporum (Tables S2, Table S3; Pel et al. 2007). The gene coding for the regulatory Rho1 GTPase subunit (TmelRhoA) was also identified (Amicucci et al. 2011).

Various $\beta$-1,3-glucan hydrolytic (or modifying) enzymes (endo- $\beta$-1,3-glucanase, transglycosidase, exo- $\beta$-1,3-glucanase) were identified (Table S2). Glycosyltransferases (e.g., members of the yeast GAS family and the A. fumigatus GEL family) are responsible for the elongation of $\beta-1,3$-glucan chains (Ragni et al. 2007). These GPI-anchored proteins (see below) are present in many yeast and fungal species and form the GH72 family in the CAZy database (http://www.cazy.org/GH72.html). The GH72 sequences can be divided into two subfamilies, which differ according to the presence (GH72+) or absence (GH72-) of a Cys-box, annotated as the X8 domain (PF07983); Ragni et al. 2007). Four members were identified in the T. melanosporum genome. One of the four members of this family belongs to group GH72+ (TmelGel4), while the other three belong to group GH72-. Only one putative $\alpha-1,3$-glucan synthase (Tmelags) was identified in T. melanosporum, as opposed to two genes in Neurospora and A. nidulans, three in A. fumigatus and five in A. niger (Table S3; Pel et al. 2007).

$\beta$-1,6-Linked glucans play a pivotal role in cell wall assembly in yeast, but are not present in several filamentous fungi (Borkovich et al. 2004; Ruiz-Herrera et al. 2008). At least four candidate genes potentially involved in $\beta$-1,6-linked glucan synthesis were identified in T. melanosporum, but they all lacked expressed sequence tag (EST) coverage in both the fruiting bodies (FB) and FLM.

\section{Other cell wall and secreted proteins}

Fifty-four putative GPI-anchored proteins were identified using the T. melanosporum protein data set (http://mycor.nancy.inra.fr/IMGC/TuberGenome/), applying the big-PI Fungal Predictor algorithm (Eisenhaber et al. 2004; Table S4) and checking for a predicted signal peptide (Bendtsen et al. 2004); among them, four had twilight zone hits (1:S 2:N and 1:S 2:S in Table S4). An 
additional step to filter out sequences with predicted transmembrane domains in the mature protein reduced the final number to 49 , which represented $0.7 \%$ of the proteome. The number of GPI proteins seems in line with that found in other fungi; for instance, 82 putative GPI proteins or $0.8 \%$ of the total were identified in the A. nidulans genome applying a similar set of algorithms (de Groot et al. 2009). Among the identified proteins are common fungal GPI proteins (see de Groot et al. 2009), i.e., all the $\beta-1,3$-glycosyltransferases found in the T. melanosporum genome (TmelGEL1, TmelGEL2, TmelGEL4, TmelGAS4); two putative glycosidases belonging to GH family 16 (Tmelcrf1, Tmelcrf2), which could be involved in cross-linking $\beta$-glucan and chitin; a putative Ecm33 protein (GSTUMT00004007001), involved in fungal cell wall organization (Pardo et al. 2004); a putative pepsin-like aspartic-type protease (GSTUMT00005184001) and a putative peptidase (GSTUMT00004908001). One hydrophobin (TmelHYD2; see below) and a protein containing a CFEM domain (TmelCFEM; see below) are also predicted to be GPI anchored. However, most of the predicted GPI proteins have unknown functions and show homology with unknown proteins in other fungal species. Expression data concerning the GPI-anchored protein subset is shown in the supplementary information (Table S5).

The cell wall-related genes identified in the T. melanosporum genome also included proteins involved in mannoprotein biosynthesis (e.g., MNN9 complex proteins) as well as enzymes involved in the processing of cell wall components, e.g., mannosidase DCW1-like, putative chitin deacetylases (CDA). In addition to the proteins involved in its synthesis and degradation, several categories of fungal cell wall proteins have been distinguished (Coronado et al. 2007; Bowman and Free 2006; de Groot et al. 2009).

Small secreted proteins (SSPs), with a putative role in the adhesion to the environment, are also present in the cell wall of filamentous fungi, i.e., hydrophobins. In the T. melanosporum genome, we identified four genes encoding proteins with the typical eight-cysteine pattern of hydrophobins, but without EST coverage. Phylogenetic analysis, in which we also included sequences belonging to a novel subclass in class I (Seidl-Seiboth et al. 2011), showed that three of the four putative hydrophobins clustered in a distinct clade (Fig. $\underline{4}$ ), while one was dispersed inside a group of sequences from Ascomycetes. Interestingly, the three corresponding genes, TmelHYD1, TmelHYD2 and TmelHYD3, are all located on the same scaffold (scaffold_3). Class II hydrophobins seems to be absent in T. melanosporum, unlike other Ascomycetes (Kubicek et al. 2008). All the putative T. melanosporum hydrophobin sequences had a predicted signal peptide, and a hydrophobin/HYDRO domain was recognized by means of InterProScan (not shown). TmelHYD3 has only six cysteine residues, suggesting that it may be a false positive or a very particular member of this family, while TmelHYD2 is predicted to be GPI-anchored. The predicted gene models were experimentally validated through TmelHYD1, TmelHYD2 and TmelHYD3 cDNA full-length sequencing, while we were unable to obtain the full-length sequence for TmelHYD4 (FS and RB, unpublished results). A GPI-anchored SSP (TmelCFEM) containing a CFEM domain (an eight-cysteine-containing domain, PF05730) was also identified. This domain is common in "fungal extracellular membrane proteins" (Kulkarni et al. 2003), but it also appears in several cell wall proteins, At least one putative chitinbinding protein (GSTUMT00010448001), containing a domain Chitin_bind_1 (PF00187), is present in T. melanosporum genome. The same domain was found also in two proteins, which have a Polysacc_deac_1 domain (TmelAGI, with three modules involved in chitin binding, and TmelPDA3). In addition, a gene (TmelPLA2), homologous to that previously identified and characterized in T. borchii (TbSP1; Soragni et al. 2001), and encoding a secreted PLA2 (phospholipase A2), which was localized in the fungal cell wall, was also found in the $T$. melanosporum genome. These two genes have the same gene structure, although the product length is 216 aa in T. melanosporum and 211 aa in T. borchii. 


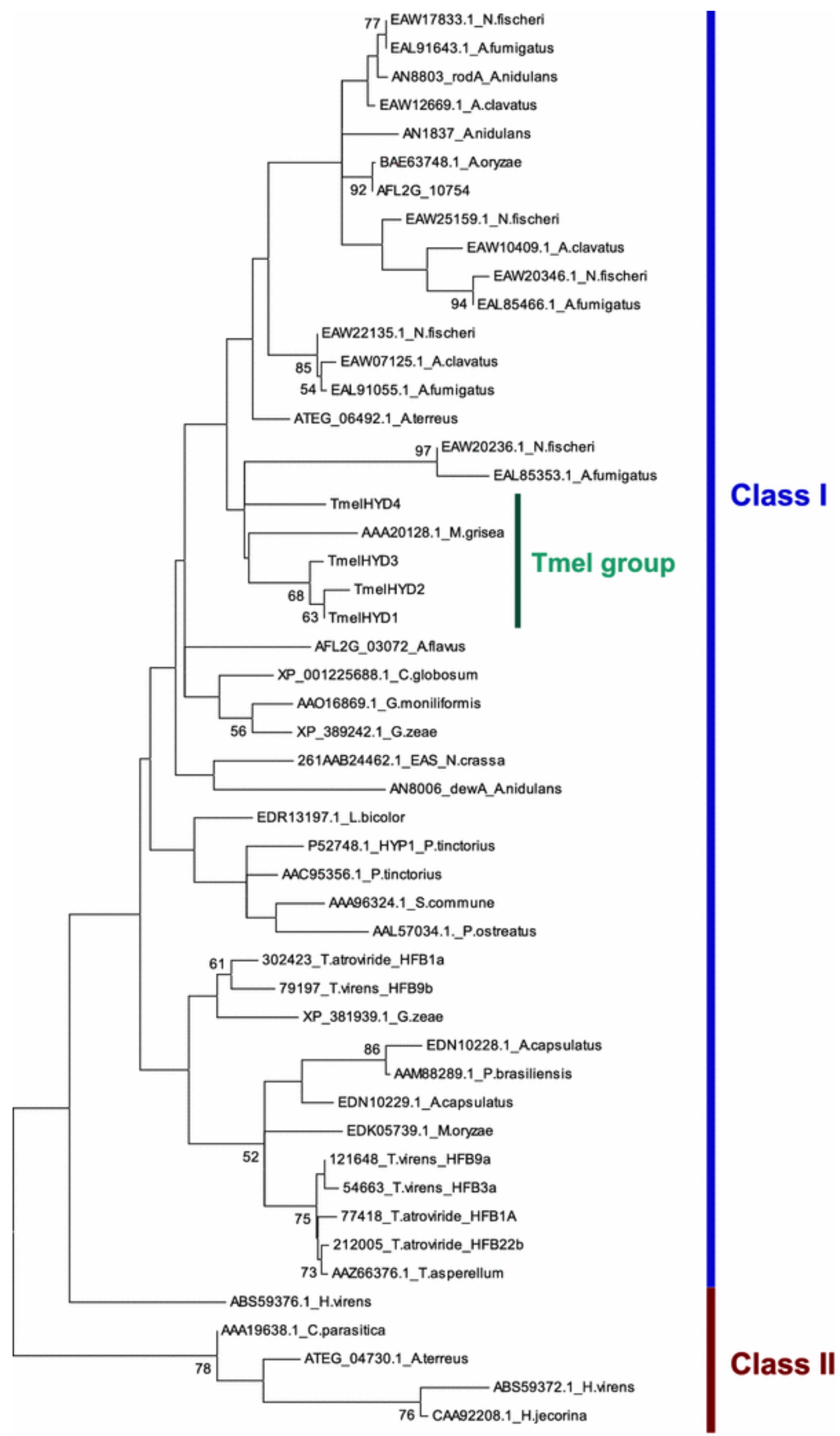


Fig. 4

Phylogenetic relationships between filamentous Ascomycetes and Basidiomycetes based on amino acid deduced sequences for the representative hydrophobin family. The sequences were aligned using Muscle and the unrooted tree was constructed using the maximum likelihood method. Numbers indicate bootstrap values and are given only for $>50 \%$. The T. melanosporum sequences analyzed in this study have been highlighted

\section{Array validation}

Whole-genome expression oligoarrays and RNA sequencing provided a first functional view of the differential expression of the identified protein-coding genes involved in the cell wall metabolism in the ECM and/or FB (fruiting body) stages (see Martin et al. 2010 for details). The complete expression data set is available as a series (accession number GSE17529) in the Gene Expression Omnibus at NCBI (http://www.ncbi.nlm.nih.gov/geo/). Briefly, 11 and 5 genes, among those included in Table S2, were expressed more than fourfold in ECM or FB relative to FLM, respectively. The TmelCHT2.1 gene was up-regulated more than fourfold in both life cycle stages (ECM and FB). The most up-regulated gene in FB versus FLM, among the cell wall-related genes, encodes a GPI-anchored protein, TmelGEL1, which is involved in $\beta$-1,3-glucan synthesis (Table S5). TmelPLA2 was one of the most up-regulated transcripts in T. melanosporum/C. avellana root tips, a result that was in agreement with previous data, which showed a role for the T. borchii homolog TbSP1 during ECM development (Miozzi et al. 2005). Surprisingly, the array expression data showed that TmelKRE6 seemed to be strongly up-regulated in the ECM stage.

In the present work, quantitative RT-PCR reactions were performed to validate the array data, considering fully established ECM. As reported in Table $\underline{1}$, we performed qRT-PCR for 29 genes. The quantitative RT-PCR confirmed the array trend for almost all of the considered genes (Table 1). Up-regulation of two chitinases, belonging to the same group, was confirmed, while the other three members in the gene family did not seem to play a role in ECM development. The unexpected high up-regulation of a gene encoding a protein involved in $\beta$-1,6-glucan (TmelKre6) metabolism was confirmed, which would seem to suggest that this enzyme could play a role in the interaction with plants. Other cell wall-associated enzymes involved in the $\beta$-1,3-glucan metabolism were also confirmed to be up-regulated (TmelEXG and TmelEXG1). Some chitin synthases (TmelCHSD,TmelCHS2, TmelCHS3, TmelCHS6), a hydrophobin (TmelHYD3) and two polysaccharide deacetylase genes (TmelPDA1 and TmelPDA2) were also confirmed. Table 1

Expression values in qRT-PCR and microarray analysis

\begin{tabular}{|c|c|c|c|c|c|c|c|}
\hline model \# & Name & $\begin{array}{c}\text { Fold } \\
\text { change } \\
\text { qRT-PCR }\end{array}$ & $\begin{array}{c}\text { Ratio } \\
\text { ECM/FL } \\
\text { M array }\end{array}$ & $\begin{array}{c}P \text { value } \\
\text { ECM/FL } \\
\text { M array }\end{array}$ & $\begin{array}{c}\text { Ratio } \\
\text { ECM/FL } \\
\text { M Solexa }\end{array}$ & $\begin{array}{c}\text { Solexa } \\
\text { ECM } \\
\text { (RPK } \\
\text { M) }\end{array}$ & $\begin{array}{c}\text { Solexa } \\
\text { FLM } \\
\text { (RPK } \\
\text { M) }\end{array}$ \\
\hline
\end{tabular}

CHSs, chitinases, $N$-acetylglucosaminidases (GH20)

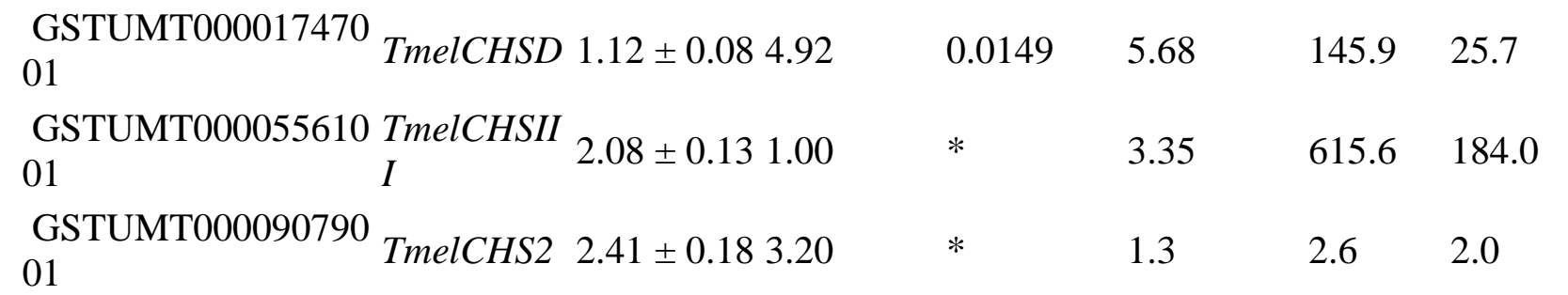




\begin{tabular}{|c|c|c|c|c|c|c|}
\hline Gene model \# & $\begin{array}{c}\text { Fold } \\
\text { change } \\
\text { qRT-PCR }\end{array}$ & $\begin{array}{c}\text { Ratio } \\
\text { ECM/FL } \\
\text { M array }\end{array}$ & $\begin{array}{c}P \text { value } \\
\text { ECM/FL } \\
M \text { array }\end{array}$ & $\begin{array}{c}\text { Ratio } \\
\text { ECM/FL } \\
\text { M Solexa }\end{array}$ & $\begin{array}{c}\text { Solexa } \\
\text { ECM } \\
\text { (RPK } \\
\text { M) }\end{array}$ & $\begin{array}{c}\text { Solexa } \\
\text { FLM } \\
\text { (RPK } \\
\text { M) }\end{array}$ \\
\hline $\begin{array}{l}\text { GSTUMT000081190 TmelCHS6 } \\
01\end{array}$ & $2.69 \pm 1.28$ & 1.01 & $*$ & 2.06 & 35.2 & 17.1 \\
\hline $\begin{array}{l}\text { GSTUMT000062290 TmelCHI } \\
01\end{array}$ & $0.68 \pm 0.50$ & 0.77 & $*$ & 0.88 & 38.3 & 43.5 \\
\hline $\begin{array}{l}\text { GSTUMT000111960 TmelCHT1 } \\
01\end{array}$ & $0.25 \pm 0.03$ & 1.49 & $*$ & 1.07 & 49.1 & 45.9 \\
\hline $\begin{array}{l}\text { GSTUMT000113260 TmelCHT2. } \\
01\end{array}$ & $\begin{array}{l}46.59 \pm 3.2 \\
7\end{array}$ & 84.95 & 0.029 & 82.50 & 16.5 & 0.2 \\
\hline $\begin{array}{l}\text { GSTUMT000115290 TmelCHT2. } \\
01\end{array}$ & $5.60 \pm 1.14$ & 12.54 & 0.00051 & 11.63 & 77.9 & 6.7 \\
\hline $\begin{array}{l}\text { GSTUMT000019750 } \\
01\end{array}$ & $0.24 \pm 0.16$ & 1.00 & $*$ & 2.71 & 1.9 & 0.7 \\
\hline $\begin{array}{l}\text { GSTUMT000068760 Tmelhex } \\
01\end{array}$ & $3.57 \pm 0.10$ & 3.52 & $*$ & 3.05 & 6.7 & 2.2 \\
\hline $\begin{array}{l}\text { GSTUMT000100840 Tmelhex2 } \\
01\end{array}$ & $2.85 \pm 1.55$ & 4.51 & 0.00214 & 5.88 & 28.8 & 4.9 \\
\hline \multicolumn{7}{|c|}{ Proteins involved in glucan metabolism } \\
\hline $\begin{array}{l}\text { GSTUMT000025530 TmelGEL1 } \\
01\end{array}$ & $0.70 \pm 0.16$ & 0.82 & $*$ & 1.13 & 0.9 & 0.8 \\
\hline $\begin{array}{l}\text { GSTUMT000108890 TmelGEL2 } \\
01\end{array}$ & $0.30 \pm 0.06$ & 1.17 & $*$ & 1.26 & 61.4 & 48.9 \\
\hline $\begin{array}{l}\text { GSTUMT000074900 TmelGEL4 } \\
01\end{array}$ & $0.32 \pm 0.13$ & 0.97 & $*$ & 2.28 & 541.9 & 237.2 \\
\hline $\begin{array}{l}\text { GSTUMT000040230 } \\
01\end{array}$ & $\begin{array}{l}0.80 \pm 0.00 \\
1\end{array}$ & 1.23 & $*$ & 0.88 & 300.0 & 338.4 \\
\hline $\begin{array}{l}\text { GSTUMT000085300 TmelEXG } \\
01\end{array}$ & $\begin{array}{l}14.26 \pm 4.6 \\
2\end{array}$ & 68.50 & 0.0134 & 37.50 & 15.0 & 0.4 \\
\hline $\begin{array}{l}\text { GSTUMT000118890 TmelEXG1 } \\
01\end{array}$ & $5.35 \pm 1.44$ & 103.65 & 0.007 & 22.00 & 4.4 & 0.2 \\
\hline $\begin{array}{l}\text { GSTUMT000021300 TmelKre6 } \\
01\end{array}$ & $\begin{array}{l}25.68 \pm 2.8 \\
4\end{array}$ & 833.40 & 0.000822 & 10.25 & 4.1 & 0.4 \\
\hline $\begin{array}{l}\text { GSTUMT000023210 } \\
01\end{array}$ & $0.50 \pm 0.12$ & 0.18 & 0.0675 & 0.27 & 1.9 & 7.1 \\
\hline $\begin{array}{l}\text { GSTUMT000126420 TmelKre9 } \\
01\end{array}$ & $0.47 \pm 0.21$ & 0.72 & $*$ & 0.71 & 34.6 & 51.6 \\
\hline $\begin{array}{l}\text { GSTUMT000063970 TmelROT1 } \\
01\end{array}$ & $0.75 \pm 0.58$ & 0.82 & $*$ & 0.98 & 23.9 & 24.5 \\
\hline $\begin{array}{l}\text { GSTUMT000025310 Tmelags } \\
01\end{array}$ & $2.92 \pm 0.89$ & 2.04 & $*$ & 1.52 & 7.0 & 4.6 \\
\hline \multicolumn{7}{|l|}{ Putative CDA, hydrophobins } \\
\hline $\begin{array}{l}\text { GSTUMT000093020 TmelPdal } \\
01\end{array}$ & $2.52 \pm 0.19$ & 2.25 & 0.00625 & 4.92 & 1141.2 & 231.8 \\
\hline
\end{tabular}




\begin{tabular}{|c|c|c|c|c|c|c|c|}
\hline Gene model \# & Name & $\begin{array}{c}\text { Fold } \\
\text { change } \\
\text { qRT-PCR }\end{array}$ & $\begin{array}{c}\text { Ratio } \\
\text { ECM/FL } \\
\text { M array }\end{array}$ & $\begin{array}{c}P \text { value } \\
\text { ECM/FL } \\
\text { M array }\end{array}$ & $\begin{array}{c}\text { Ratio } \\
\text { ECM/FL } \\
\text { M Solexa }\end{array}$ & $\begin{array}{c}\text { Solexa } \\
\text { ECM } \\
\text { (RPK } \\
\text { M) }\end{array}$ & $\begin{array}{c}\text { Solexa } \\
\text { FLM } \\
\text { (RPK } \\
\text { M) }\end{array}$ \\
\hline $\begin{array}{l}\text { GSTUMT000030320 } \\
01\end{array}$ & TmelPda2 & $0.10 \pm 0.08$ & 0.81 & $*$ & 0.83 & 70.4 & 85.0 \\
\hline $\begin{array}{l}\text { GSTUMT000042100 } \\
01\end{array}$ & TmelPda3 & $3.40 \pm 0.10$ & 4.65 & $*$ & 1.65 & 99.3 & 60.1 \\
\hline $\begin{array}{l}\text { GSTUMT000124430 } \\
01\end{array}$ & TmelHYD1 & $0.03 \pm 0.38$ & 0.55 & $*$ & 0.00 & 0.0 & 0.5 \\
\hline $\begin{array}{l}\text { GSTUMT000068640 } \\
01\end{array}$ & TmelHYD2 & $0.12 \pm 0.10$ & 0.15 & 0.0195 & 0.42 & 1.9 & 4.5 \\
\hline $\begin{array}{l}\text { GSTUMT000124440 } \\
01\end{array}$ & TmelHYD3 & $2.07 \pm 0.79$ & 2.25 & $*$ & 1.80 & 1.8 & 1.0 \\
\hline $\begin{array}{l}\text { GSTUMT000031020 } \\
01\end{array}$ & TmelHYD4 & $0.11 \pm 0.03$ & 10.31 & $*$ & 0.37 & 9.3 & 25.3 \\
\hline
\end{tabular}

Solexa data have been reported as RPKM (Reads Per kb per Million reads). Genes only slightly regulated/not regulated but with interesting function were also selected for validation

$E C M$ ectomycorrhizae, FLM free-living mycelium

$P$ values for genes that are significantly differentially regulated are shown. Genes that are not significantly differentially regulated are indicated by an asterisk

\section{Discussion}

In this work, in silico analysis of the T. melanosporum genome has been exploited to obtain detailed knowledge on cell wall-related proteins (i.e., enzymes and regulators involved in cell wall dynamics), and to identify several members of the gene families (i.e., chitin synthases, chitinases).

The analysis of proteins involved in the synthesis and remodeling of the cell wall from $T$. melanosporum revealed the existence of important similarities in this class of proteins with other fungal classes, including both yeast and filamentous fungi. It is worth that the enzymes involved in the degradation/remodeling of the cell wall seem to be less abundant than in other filamentous fungi, while the essential growth-related genes, such as CHS genes, seem to be in a similar number to other fungi. A comparison with a reference set of five Ascomycetes (S. cerevisiae, N. crassa, M. grisea, B. cinerea and Aspergillus nidulans; see Table S2) has shown that: (1) 2 genes are Tuber orthologs ('best reciprocal hits'; BRHs) of yeast genes without a BRH in the other reference Ascomycetes; (2) 50 genes (6 of which were found in Tuber, but not in the reference Ascomycetes) are only found in filamentous fungi. It should be noted that three members of group (2), which are specific for Tuber, belong to the CDA (chitin deacetylase) group. About 80-90\% of the annotated genes involved in fungal cell wall metabolism were covered by ESTs.

\section{Expression of cell wall genes in the symbiotic stage}

As a symbiotic fungus, Tuber interacts with plants and obtaining a better understanding of this process is, therefore, important to understand the mechanism involved in symbiosis development. Transcriptomic analyses (cDNA arrays and RNAseq), performed as part of the genome sequencing 
project (Martin et al. 2010), have allowed us to reveal the regulation of some of the annotated genes during the three stages of the truffle life cycle. Solexa data gave a good correspondence with the results from microarray experiment, and qRT-PCR experiments confirmed the "regulation trend" for the majority of the genes. Differences among the gene expression data obtained with the different techniques are generally present when array data are not significant (with a $P$ value $>0.05$ ), but they can also be explained by the variability in the biological material sampling.

We have focused on the symbiotic stage. During the switch from vegetative growth as filamentous mycelium toward the formation of ECM, which requires mutual recognition between the fungus and the host plant, the mycelium undergoes morphogenetic changes. These lead to the formation of symbiotic structures, such as the two fungal pseudo-tissues present in an ECM, the mantle and the Hartig net, in which adhesion events occur. During the establishment of symbiosis, the fungus has to change the apical growth to proliferate inside the root cells, producing novel morphologies, as aggregated and labyrinthine hyphae. It could be expected that the changes in growth, associated with ECM development, are accompanied by changes in cell wall transcript patterns. Fungal cell wall proteins (i.e., hydrophobins, adhesins and mannoproteins), which have been suggested to play a role in hyphal aggregation and to restructure the plant/fungus interface, have already been reported as being induced during symbiotic interaction (Martin et al. 1999). Hydrophobins, smallsized secreted proteins that are unique to filamentous fungi, are associated with the outer surface of the cell wall, where they mediate the interaction between the fungus and the environment (Whiteford and Spanu 2002). Our results showed that one of the four identified hydrophobin genes (TmelHYD3) had a slight up-regulation (about twofold) in ECM, with respect to FLM, in addition to two genes, whose products belong to the putative CDA (chitin deacetylase) group (TmelPDA3 and TmelPDA1). Six genes encoding putative polysaccharide deacetylase genes, including putative chitin deacetylases (CDA), were identified in the T. melanosporum genome. The two up-regulated genes in truffle ECMs encode two secreted proteins that contain a Poly_deac_1 domain and show similarity with microbial xylanases/deacetylases. Two biological roles have been suggested for fungal CDAs: an involvement in cell wall formation (converting chitin into chitosan); possibly, deacetylation of chitin oligomers and reduction of their elicitor activity during plant-pathogen interactions, as proposed in Colletotrichum lindemuthianum (Tsigos and Bouriotis 1995; Tsigos et al. 2000). The presence of a chitin-binding domain in TmelPDA3 should also be mentioned. A role for chitin-binding proteins has been proposed in pathogenic fungi, protecting the fungal cell wall from chitinases produced by host plants (van den Burg et al. 2006). Surprisingly, our results showed that one of the most up-regulated genes (TmelKre6) in ECM is an enzyme that is involved in $\beta-1,6-$ glucan synthesis. The synthesis of $\beta-1,6$-glucans is a complex process that involves several enzymes with different cellular locations: endoplasmic reticulum (ER), Golgi, plasma membrane and cell wall. In S. cerevisiae, where the synthesis mechanism was discovered, ten different enzymes were identified as being involved in this process (Shahinian and Bussey 2000). S. cerevisiae Kre6 is a type II membrane protein with amino acid sequence homology with glycoside hydrolase and it is essential for $\beta$-1,6-glucan synthesis, as revealed by the mutant phenotype, but its biochemical function is still unknown (Kurita et al. 2011). Kre6 was first reported as a Golgi protein, but has recently been located mostly in the ER and also in a small but significant portion, in the secretory vesicle-like compartment and plasma membrane (Kurita et al. 2011). Four homologous genes have been found in the T. melanosporum genome: TmelKre6, TmelKre9, TmelROT1 and TmelSKN1. Immunolabeling experiments have failed to detect $\beta-1,6$-glucans in the truffle cell wall. However, since no biochemical analysis has been performed on truffle cell walls, we cannot exclude that this polysaccharide component has different structural features from the yeast component, which are not recognized by the antibody. $\beta-1,6-$ Glucans have not been found in several filamentous fungi, such as the ascomycetous fungi Neurospora and A. fumigatus (Borkovich et al. 2004). Proteins reported as functioning in the ER are present in Neurospora and S. cerevisiae, while the genes KRE6 and SKN1 are present in A. fumigatus, but not in Neurospora (Borkovich et al. 2004). On the other 
hand, $\beta$-1,6-glucans have been detected in the cell wall of the basidiomycete Ustilago maydis, although the in silico analysis of its genome has only revealed the presence of six of the genes identified in yeast (Aimanianda et al. 2009). Interestingly, a novel function has been proposed for the SKN1 gene, which was previously essentially considered a KRE6 homolog involved in $\beta-1,6-$ glucan synthesis. It has been demonstrated that in yeast, $S K N 1$ is implicated in sphingolipid biosynthesis (Thevissen et al. 2005). The strong up-regulation of TmelKre6 in truffle ECM suggests that its product could play a role in the interaction with the plant, although its function in this context is still unknown.

Several other enzymes involved in cell wall modification are up-regulated in the symbiotic stage, suggesting a role during the morphogenetic transitions from FLM to ECM formation. There are two chitinases (TmelCHT2.1 and TmelCHT2.2) among the most up-regulated genes, and these might be paralogous inside chitinase group B. These proteins, together with the enzymes involved in the $\beta$ 1,3-glucan metabolism (i.e., glucanases, TmelEXG and TmelEXG1), could play a role in cell wall remodeling, which is probably essential during the switch from the free-living to the symbiotic status, where the mantle and Hartig net are formed.

Two chitin synthase genes (TmelCHSD and TmelCHS2) are also slightly up-regulated in symbiotic structures. The up-regulation in ECM of genes encoding enzymes involved in the cell wall metabolism suggest their involvement in fungal cell wall remodeling, which involves both synthesis and degradation processes, during the growth and the branching of the hyphae hosted in the root.

\section{Conclusions}

The availability of the T. melanosporum genome has made it possible to carry out a detailed in silico analysis of cell wall-related genes, which are crucial to understand the growth processes and mechanisms that are at the base of cell-to-cell interactions during the symbiotic phase. The annotation results have given us a glimpse into the cell wall metabolism, thanks to the identification of members of several gene families. All these proteins could play a role in fungal cell wall remodeling during the switch from the free living to the symbiotic state. The results highlight some of the molecular processes that influence truffle growth and its interaction with the host. However, an ECM is a complex organ that is not only formed by two individuals (plant/fungus), but also by two fungal pseudo-tissues, which are easily distinguishable under the microscope: the mantle, which develops outside the root, and the Hartig net, which is formed between the root cells, and is considered the site where nutritional exchanges take place between the partners. The objectives of future studies will be to verify the "tissue" specificity in an ECM, i.e., verifying the transcript profiles associated with the two fungal compartments (mantle and Hartig net).

\section{Acknowledgments}

The Tuber genome sequencing project is a collaborative effort involving the Génoscope-CEA and the Tuber Genome Consortium. The authors would like to thank Emmanuelle Morin and Stefano Ghignone for the bioinformatics support; Elisa Zampieri for the help during the preparation of the manuscript, Michele Buffalini, Paola Ceccaroli and Shwet Kamal for the annotation of some genes cited in the paper. This research was funded by PRIN2007; FS's fellowship PhD was funded by Università di Torino. 


\section{Supplementary material}

Supplementary Data are available at CURRENT GENETICS Online.

\section{References}

1. Aimanianda V, Bayry J, Bozza S, Kniemeyer O, Perruccio K, Elluru SR, Clavaud C, Paris S, Brakhage AA, Kaveri SV, Romani L, Latge JP (2009) Surface hydrophobin prevents immune recognition of airborne fungal spores. Nature 460:1117-1121

2. Amicucci A, Balestrini R, Kohler A, Barbieri E, Saltarelli R, Faccio A, Roberson RW, Bonfante P, Stocchi V (2011) Hyphal and cytoskeleton polarization in Tuber melanosporum: a genomic and cellular analysis. Fungal Genet Biol 48:561-572

3. Balestrini R, Hahn MG, Bonfante P (1996) Location of cell-wall components in ectomycorrhizae of Corylus avellana and Tuber magnatum. Protoplasma 191:55-69

4. Balestrini R, Mainieri D, Soragni E, Garnero L, Rollino S, Viotti A, Ottonello S, Bonfante P (2000) Differential expression of chitin synthase III and IV mRNAs in ascomata of Tuber borchii Vittad. Fungal Genet Biol 31:219-232

5. Balestrini R, Bianciotto V, Bonfante P (2011) Mycorrhizae in: Huang, Li, Sumner (eds) Handbook of Soil Sciences, Volume I. Taylor and Francis Group, Boca Raton, pp 24/29$24 / 40$

6. Bendtsen JD, Nielsen H, von Heijne G, Brunak S (2004) Improved prediction of signal peptides: SignalP 3.0. J Mol Biol 340:783-795

7. Borgia PT, Iartchouk N, Riggle PJ, Winter KR, Koltin Y, Bulawa CE (1996) The chsB gene of Aspergillus nidulans is necessary for normal hyphal growth and development. Fungal Genet Biol 20:193-203

8. Borkovich KA, Alex LA, Yarden O et al (2004) Lessons from the genome sequence of Neurospora crassa: tracing the path from genomic blueprint to multicellular organism. Microbiol Mol Biol Rev 68:1-108

9. Bowman SM, Free SJ (2006) The structure and synthesis of the fungal cell wall. BioEssays 28:799-808

10. Brendel V, Bucher P, Nourbakhsh IR, Blaisdell BE, Karlin S (1992). "Methods and algorithms for statistical analysis of protein sequences." Proceedings of the National Academy of Sciences of the United States of America 89 (6) (March 15):2002-2006

11. Cantarel BL, Coutinho PM, Rancurel C, Bernard T, Lombard V, Henrissat B (2009) The Carbohydrate-Active EnZymes database (CAZy): an expert resource for glycogenomics. Nucleic Acids Res 37:D233-D238

12. Chang S, Puryear J, Cairney J (1993) A simple and efficient method for isolating RNA from pine trees. Plant Mol Biol Rep 11:113-116

13. Choquer M, Boccara M, Goncalves IR, Soulié M-C, Vidal-Cros A (2004) Survey of the Botrytis cinerea chitin synthase multigenic family through the analysis of six euascomycetes genomes. Eur J Biochem 271:2153-2164

14. Coronado JE, Mneimneh S, Epstein SL, Qiu WG, Lipke PN (2007) Conserved processes and lineage-specific proteins in fungal cell wall evolution. Eukaryot Cell 6:2269-2277

15. Cserzo M, Eisenhaber F, Eisenhaber B, Simon I (2004) TM or not TM: transmembrane protein prediction with low false positive rate using DAS-TMfilter. Bioinformatics 20:136137

16. de Groot PWJ, Brandt BW, Horiuchi H, Ramd AFJ, de Koster CG, Klis FM (2009) Comprehensive genomic analysis of cell wall genes in Aspergillus nidulans. Fungal Genet Biol 46:S72-S81 
17. Denoeud F, Aury JM, Da Silva C, Noel B, Rogier O, Delledonne M, Morgante M, Valle G, Wincker P, Scarpelli C, Jaillon O, Artiguenave F (2008) Annotating genomes with massivescale RNA sequencing. Genome Biol 9:R175

18. Duo-Chuan L (2006) Review of fungal chitinases. Mycopathologia 161:345-360

19. Edgar RC (2004) MUSCLE: multiple sequence alignment with high accuracy and high throughput. Nucleic Acids Res 32:1792-1797

20. Eisenhaber B, Bork P, Eisenhaber F (1999) Prediction of potential GPI-modification sites in proprotein sequences. J Mol Biol 292:741-758

21. Eisenhaber B, Schneider G, Wildpaner M, Eisenhaber F (2004) A sensitive predictor for potential GPI lipid modification sites in fungal protein sequences and its application to genome-wide studies for Aspergillus nidulans, Candida albicans, Neurospora crassa, Saccharomyces cerevisiae and Schizosaccharomyces pombe. J Mol Biol 337:243-253

22. Gruber S, Vaaje-Kolstad G, Matarese F, López-Mondéjar R, Kubicek CP, Seidl-Seiboth V (2011) Analysis of subgroup $C$ of fungal chitinases containing chitin-binding and LysM modules in the mycoparasite Trichoderma atroviride. Glycobiology 21:122-133

23. Käll L, Krogh A, Sonnhammer ELL (2004) A combined transmembrane topology and signal peptide prediction method. J Mol Biol 338:1027-1036

24. Karlsson M, Stenlid J (2008) Comparative evolutionary histories of the fungal chitinase gene family reveal non-random size expansions and contractions due to adaptive natural selection. Evol Bioinform 4:47-60

25. Kubicek C, Baker S, Gamauf C, Kenerley C, Druzhinina I (2008) Purifying selection and birth-and-death evolution in the class II hydrophobin gene families of the ascomycete Trichoderma/Hypocrea. BMC Evol Biol 8:4

26. Kulkarni RD, Kelkar HS, Dean RA (2003) An eight-cysteine-containing CFEM domain unique to a group of fungal membrane proteins. TIBS 28:118-121

27. Kurita T, Noda Y, Takagi T, Osumi M, Yoda K (2011) Kre6 protein essential for yeast cell wall $\beta$-1,6-glucan synthesis accumulates at sites of polarized growth. J Biol Chem 286:7429-7438

28. Laurent P, Voiblet C, Tagu D, de Carvalho D, Nehls U, De Bellis R, Balestrini R, Bauw G, Bonfante P, Martin F (1999) A novel class of ectomycorrhiza-regulated cell wall polypeptides in Pisolithus tinctorius. Mol Plant Microbe Interact 12:862-871

29. Lesage G, Bussey H (2006) Cell wall assembly in Saccharomyces cerevisiae. Microbiol Mol Biol Rev 70:317-343

30. Martin F, Laurent P, de Carvalho D, Voiblet C, Balestrini R, Bonfante P, Tagu D (1999) Cell wall proteins of the ectomycorrhizal basidiomycete Pisolithus tinctorius: identification, function, and expression in symbiosis. Fungal Genet Biol 27:161-174

31. Martin F, Kohler A, Murat C, Balestrini R et al (2010) Périgord black truffle genome uncovers evolutionary origins and mechanisms of symbiosis. Nature 464:1033-1038

32. Martin-Urdiroz M, Roncero MIG, Gonzalez-Reyes JA, Ruiz-Roldan C (2008) ChsVb, a Class VII chitin synthase involved in septation, is critical for pathogenicity in Fusarium oxysporum. Eukaryot Cell 7:112-121

33. Miozzi L, Balestrini R, Bolchi A, Novero M, Ottonello S, Bonfante P (2005) Phospholipase A2 up-regulation during mycorrhiza formation in Tuber borchii. New Phytol 167:229-238

34. Montijn RC, Vink E, Muller WH, Verkleij AJ, Van Den Ende H, Henrissat B, Klis FM (1999) Localization of synthesis of $\beta$-1,6-glucan in Saccharomyces cerevisiae. J Bacteriol $181: 7414-7420$

35. Ooi HS, Kwo CY, Wildpaner M, Sirota FL, Eisenhaber B, Maurer-Stroh S, Wong WC, Schleiffer A, Eisenhaber F, Schneider G (2009) ANNIE: integrated de novo protein sequence annotation. Nucleic Acids Res 37:435-440 
36. Pardo M, Monteoliva L, Vazquez P, Martinez R, Molero G, Nombela C, Gil C (2004) PST1 and ECM33 encode two yeast cell surface GPI proteins important for cell wall integrity. Microbiology 150:4157-4170

37. Pel HJ, De Winde JH, Archer DB et al (2007) Genome sequencing and analysis of the versatile cell factory Aspergillus niger CBS 51388. Nat Biotech 25:221-231

38. Ragni E, Fontaine T, Gissi C, Latgè JP, Popolo L (2007) The Gas family of proteins of Saccharomyces cerevisiae: characterization and evolutionary analysis. Yeast 24:297-308

39. Ruiz-Herrera J, Ortiz-Castellanos L, Martínez A-I, León-Ramírez C, Sentandreu R (2008) Analysis of the proteins involved in the structure and synthesis of the cell wall of Ustilago maydis. Fungal Genet Biol 45:S71-S76

40. Seidl V (2008) Chitinases of filamentous fungi: a large group of diverse proteins with multiple physiological functions. Fungal Biol Rev 22:36-42

41. Seidl-Seiboth V, Gruber S, Sezerman U, Schwecke T, Albayrak A, Neuhof T, Von Döhren H, Baker SE, Kubicek CP (2011) Novel hydrophobins from Trichoderma define a new hydrophobin subclass: protein properties, evolution, regulation and processing. J Mol Evol 72:339-351

42. Shahinian S, Bussey H (2000) $\beta-1,6-$ Glucan synthesis in Saccharomyces cerevisiae. Mol Microbiol 35:477-489

43. Soragni E, Bolchi A, Balestrini R, Gambaretto C, Percudani R, Bonfante P, Ottonello S (2001) A nutrient-regulated, dual localization phospholipase A2 in the symbiotic fungus Tuber borchii. EMBO J 20:5079-5090

44. Tagu D, De Bellis R, Balestrini R, de Vries OMH, Piccoli G, Stocchi V, Bonfante P, Martin F (2001) Immunolocalization of hydrophobin HYDPt-1 from the ectomycorrhizal basidiomycete Pisolithus tinctorius during colonization of Eucalyptus globulus roots. New Phytol 149:127-135

45. Takeshita N, Yamashita S, Ohta A, Horiuchi H (2006) Aspergillus nidulans class V and VI chitin synthases CsmA and $\mathrm{CsmB}$, each with a myosin motor-like domain, perform compensatory functions that are essential for hyphal tip growth. Mol Microbiol 59:1380 1394

46. Tamura K, Peterson D, Peterson N, Stecher G, Nei M, Kumar S (2011) MEGA5: molecular evolutionary genetics analysis using maximum likelihood, evolutionary distance, and maximum parsimony methods. Mol Biol Evol 28:2731-2739

47. Thevissen K, Idkowiak-Baldys J, Im YJ, Takemoto J, François IE, Ferket KK, Aerts AM, Meert EM, Winderickx J, Roosen J, Cammue BP (2005) SKN1, a novel plant defensinsensitivity gene in Saccharomyces cerevisiae, is implicated in sphingolipid biosynthesis. FEBS Lett 579:1973-1977

48. Treitschke S, Doehlemann G, Schuster M, Steinberg G (2010) The myosin motor domain of fungal chitin synthase $\mathrm{V}$ is dispensable for vesicle motility but required for virulence of the maize pathogen Ustilago maydis. Plant Cell 22:2476-2494

49. Tsigos I, Bouriotis V (1995) Purification and characterization of chitin deacetylase from Colletotrichum lindemuthianum. J Biol Chem 270:26286-26291

50. Tsigos I, Martinou A, Kafetzopoulos D, Bouriotis V (2000) Chitin deacetylases: new, versatile tools in biotechnology. Trends Biotech 18:305-312

51. Tusnády GE, Simon I (2001) The HMMTOP transmembrane topology prediction server. Bioinformatics 17:849-850

52. van den Burg HA, Harrison SJ, Joosten MH, Vervoort J, de Wit PJ (2006) Cladosporium fulvum Avr4 protects fungal cell walls against hydrolysis by plant chitinases accumulating during infection. Mol Plant Microbe Interact 19:1420-1430

53. Weber I, Aßmann D, Thines E, Steinberg G (2006) Polar localizing class v myosin chitin synthases are essential during early plant infection in the plant pathogenic fungus Ustilago maydis. Plant Cell 18:225-242 
54. Whiteford JR, Spanu PD (2002) Hydrophobins and the interactions between fungi and plants. Mol Plant Pathol 3:391-400

55. Zampieri E, Balestrini R, Kohler A, Abbà S, Martin F, Bonfante P (2011) The Perigord black truffle responds to cold temperature with an extensive reprogramming of its transcriptional activity. Fungal Genet Biol 48:585-591 